\title{
A method to reduce soft tissue artifact in motion analysis using the dependency between the anatomical landmark and skin marker displacements
}

\author{
Taebeum Ryu ${ }^{1}$, Hwa Soon Choi $^{2}$, Min K. Chung ${ }^{2}$ \\ ${ }^{1}$ Department of Industrial \& Management Engineering, Hanbat National University, Deajeon, \\ Korea \\ ${ }^{2}$ Department of Industrial \& Management Engineering, POSTECH, Pohang, Korea \\ tbryu@hanbat.ac.jp
}

\begin{abstract}
This study proposed a method to reduce the motion analysis error due to soft tissue artifact using a systematic pattern between the displacement of anatomical landmark and skin marker. A validation experiment to test the feasibility of the method was performed in which a female wearing an external fixator on the right shank. The proposed method reduced the error of the shank motion more than the comparable previous method, and the error reductions for some of kinematic variables were statistically significant.
\end{abstract}

Keywords: Motion analysis, Soft tissue artifact, Anatomical landmark calibration, Skin marker displacement

\section{Introduction}

Skin marker-based stereophotogrammetry is most frequently used to analyze human motions for its advantages in safety and usability. But, motion analysis based on stereophotogrammetry has been known to have fairly large error due to the deformation of soft tissues such as skin and muscle, which is called a soft tissue artifact (STA). The motion analysis error due to STA was reported to range from 10 to $20^{\circ}$ and especially significant in the aspects of ab/adduction and in/external rotation $[1,2,3]$.

Lucchetti et al. [4] proposed so-called dynamic anatomical landmark (AL) calibration to identify $\mathrm{AL}$ positions in an ad hoc motion. Instead of assessing the STA effect with skin marker displacement relative to the underlying bone, this method innovatively used the relative movement of ALs in the reference coordinate frame defined by STA-affected skin markers, which is usually referred to as technical coordinate frame (TCF). Then they modeled the displacement of ALs with joint angles to compensate AL positions relative to TCF in a motor task. But, the previous dynamic calibration has some inefficiency in the repetitive calculations of joint angles for the compensation of the $\mathrm{AL}$ displacement.

The present study proposed an alternative to the $\mathrm{AL}$ displacement modeling with joint angle of the previous dynamic calibration method. This study assumed that the $\mathrm{AL}$ displacement is also associated with the displacement of some skin markers in the identical TCF and attempted to model the dependency between them. Because the calculation of the skin marker displacement is simple, $\mathrm{AL}$ displacement compensation using skin marker displacement will take much less time and effort than using joint angles. The feasibility of the proposed method was tested by analyzing knee motions of a patient wearing an external fixator on the shank, and its performance was compared with the previous dynamic calibration method using joint angle.

\section{STA compensation method}

The dynamic calibration identifies $\mathrm{AL}$ position of a segment with the skin marker cluster of a neighbor segment which is not affected by STA during an ad hoc motion. The STA of a segment mostly originates from the rotation of the adjacent joints [1]. Thus, during a joint rotation with an adjacent joint fixed, STA affects the segment near the rotated joint, but not one far away from it. Based on this idea, AL positions of the segment near the rotated joint are identified with their local coordinate in the reference of the TCF of the STA-free segment during the ad hoc motion.

From the positions of ALs on a segment identified during an ad hoc motion, AL displacement is calculated in the reference of a TCF on the segment. During an ad hoc motion, because skin marker cluster on a segment near the rotated joint deforms due to STA, the pose of a TCF defined by the cluster changes relative to the underlying bone. Thus, as shown in Figure 1 [a], if the local coordinate of an AL is determined in the TCF defined by $S_{1}, S_{2}$ and $S_{3}$ in a static posture, a difference $\left(\Delta r_{a}\right)$ will be occurred between the obtained $A L$ positions in the ad hoc motion and ones reconstructed by its local coordinate in a static posture (Figure 1[b]).

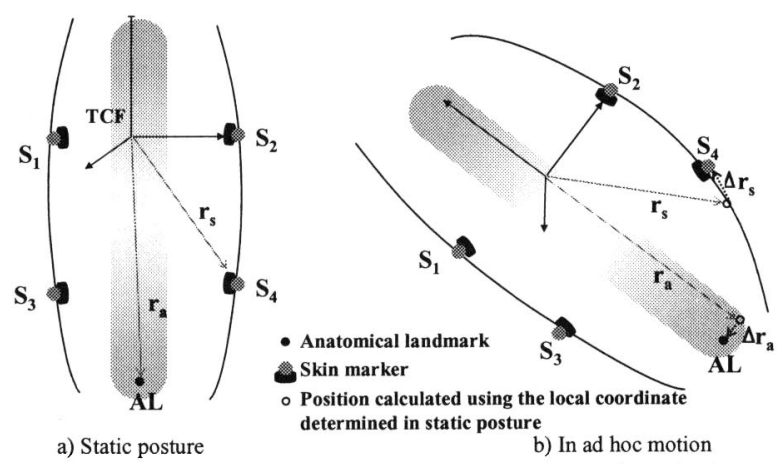

Figure 1. Displacements of AL and a skin marker $\left(\mathrm{S}_{4}\right)$ to a TCF defined by $S_{1}-S_{3}$ 
Likewise, the displacement of some skin markers other than ones used to define a TCF can be calculated in the reference of a TCF. While STA has a systematic pattern with regard to a motor task, its effect on each marker differs depending on their position. As shown in Figure 1, if the local coordinate of an additional skin marker is determined in the TCF of $S_{1}, S_{2}$ and $S_{3}$, there will be also a difference $\left(\Delta r_{s}\right)$ between its measured positions during the motion and ones reconstructed by its local coordinate.

The proposed method finds a dependency between the displacements of AL and skin marker and models it in a linear form. The proposed method determines an axial component of a skin marker displacement highly correlated with each axial component of an AL displacement using a correlation analysis. Then each component of an AL displacement is modeled with the correlated component of a skin marker displacement in a simple regression form.

During a motor task $\mathrm{AL}$ positions are compensated by using the developed AL displacement models. At each frame of motion, the skin marker displacement in the reference of a TCF is calculated, then AL displacement in the TCF is estimated with the developed $A L$ displacement model. The $\mathrm{AL}$ position is adjusted by adding the estimated AL displacement to the AL local coordinate in the TCF originally determined in a static posture.

\section{Validation experiment}

A motion measurement system with six cameras (Falcon, MotionAnalysis) was used to measure lower extremity motions (sampling frequency $60 \mathrm{~Hz}$, measurement volume $4 \times 3 \times 2 \mathrm{~m}$ ). One female patient wearing an external bony fixator on the right shank participated in the experiment. The height, weight and age of the patient were $1.63 \mathrm{~m}, 56 \mathrm{~kg}$ and 42 years. The participants signed an informed consent. The motion measurement was conducted on the day before the external fixator was removed. The patient performed three motor tasks: sitting static posture and two set of repeated knee motions. Sitting posture was conducted for anatomical calibration, the first knee motions for $\mathrm{AL}$ displacement modeling, and the second for analyzing motions.

Reflective markers (20 mm diameter spherical balls) were placed on the shank of the patient. Five (M1 - M5) and three markers (E1 - E3) were located on the shank and the external fixator, respectively (Figure 2). To test the feasibility of the proposed method in various marker arrangements, two marker clusters (M1, M2, M5 and M1, M2, M3) were used in the shank motion analysis, respectively. The markers on the fixator were used to analyze the skeletal movement as a gold standard. In addition, $\mathrm{AL}$ positions rigid to the tibia/fibula are identified using the fixator markers during knee motions instead of using the dynamic calibration method.

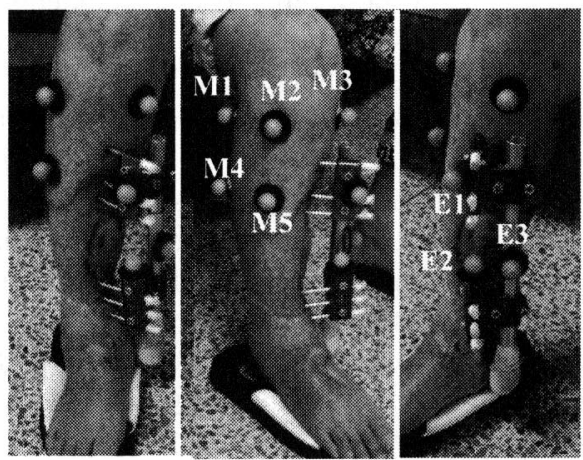

Figure 2. Marker placement

Anatomical coordinate frame of the shank was defined with lateral and medial tibial condyles (LC, MC), and lateral and medial malleolus (LM and MM) [5]. AL calibration was performed in a sitting static posture using a pointer on which two markers were mounted with a known distance. The position of LC, MC, LM and MM was measured by pointing the tip of the pointer onto the corresponding palpable ALs, meanwhile the markers on the shank and the fixator were also measured.

To identify the AL positions from the marker clusters on the shank and the fixator during motion, AL position relative to each marker cluster were determined through geometric calculation. Two TCFs $\left(\mathrm{TCF}^{1}\right.$ and $\left.\mathrm{TCF}^{2}\right)$ were defined with the two shank marker clusters, respectively, and the local coordinate of each $\mathrm{AL}$ was determined in each TCF. Likewise, the coordinate frame of the fixator was defined with the markers of E1, E2 and E3, and the local coordinate of each AL were also determined in the frame.

During motion the pose of the shank TCFs was estimated using the Singular Value Decomposition algorithm [6]. The position vector and orientation matrix of each TCF was obtained from the transformation matrix, which estimated by the algorithm, between the local coordinate of the three relevant skin markers in the TCF and their global positions.

The pose of fixator coordinate frame was also estimated in the same way from the transformation matrix between the local coordinate of the fixator markers in the frame and their global positions. Using the pose of the coordinate frame (TCFs or fixator frame) and relevant local coordinate of ALs, the pose of anatomical coordinate frame of the shank was calculated.

The displacements of ALs and a skin marker were calculated in the reference of the two shank TCFs using the recording in the first knee motions. AL positions were reconstructed using the fixator pose and the relevant AL local coordinates. Then the difference was calculated between the local coordinate of the reconstructed ALs in each TCF and ones determined in the sitting static posture Likewise, the displacement of skin marker M4 was calculated subtracting its local coordinate in each TCF determined in the static posture from the measured ones. 
The dependency between the displacements of ALs and skin marker M4 was modeled in a linear form. To compare the proposed method and the previous dynamic calibration, moreover, AL displacement was modeled with the knee rotation angles.

The AL positions during the second knee motion were estimated with the developed AL displacement model. At each frame of the motion, AL displacements were estimated using the developed models and the displacement of M4, and the local coordinate of ALs in each TCF determined in the static posture was adjusted by the relevant AL displacement.

\section{Results}

There was a dependency between the displacements of the ALs and the skin marker M4 in the corresponding TCFs during the first set of knee motion. One component of M4 displacements highly correlated with each component of AL displacements was identified. Most AL displacements had a high dependency with at least one of the three axial components of the displacements of M4.

A simple regression model for each axial component of $\mathrm{AL}$ displacements was made with the axial component of the skin marker displacement which had the highest correlation coefficient with it. Of a total of 24 regression models for AL displacements, 18 models had $R^{2}$ values higher than 0.5 .

The rotational and translational errors relative to the gold standard are shown in Figure 3 as obtained with (using skin marker displacement and rotation angle) and without $\mathrm{AL}$ compensation during the second knee motions. Regardless of marker clusters used in motion analysis, AL compensation methods had smaller errors than without compensation with respect to the rotation and translation of the shank. The results of the Student-Newman-Keuls (SNK) test about the errors for the three methods are shown in Figure 4 at $\alpha=0.05$. The AL compensation with skin marker displacement had significantly smaller $(39-83 \%)$ errors than without compensation in all kinematic variables for the two marker clusters. Moreover, it had significantly smaller $(25-58 \%)$ errors than the compensation with rotation angles in X-, Z-rotation and most translational motions except along $\mathrm{X}$ - and $\mathrm{Y}$-axis for marker cluster 1 , where no significant difference was found.

\section{Discussion and conclusions}

Both AL compensations (with skin marker displacement and rotation angle) were effective in reducing the STA errors. In the validation experiment, all the rotational and translational errors were significantly reduced using AL compensation with skin marker displacement by $40-80 \%$ with respect to without compensation. In addition, most of the rotational and translational errors were significantly reduced using $\mathrm{AL}$ compensation with rotational angle by $30-70 \%$.

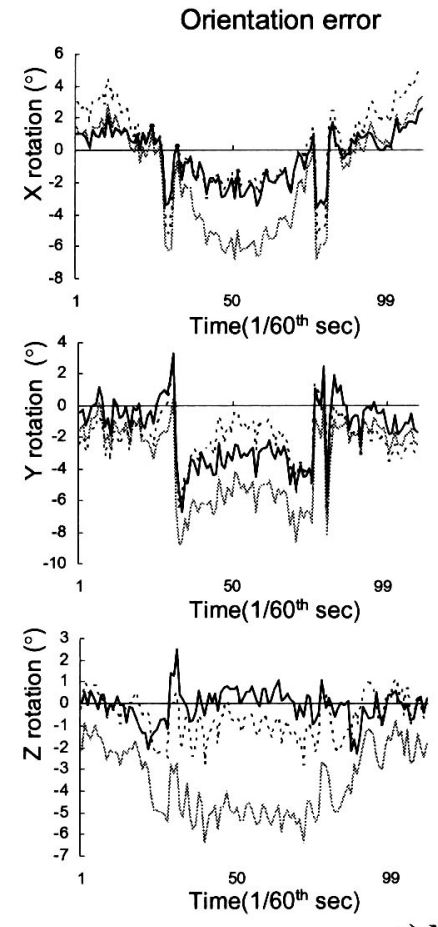

a) Marker cluster 1
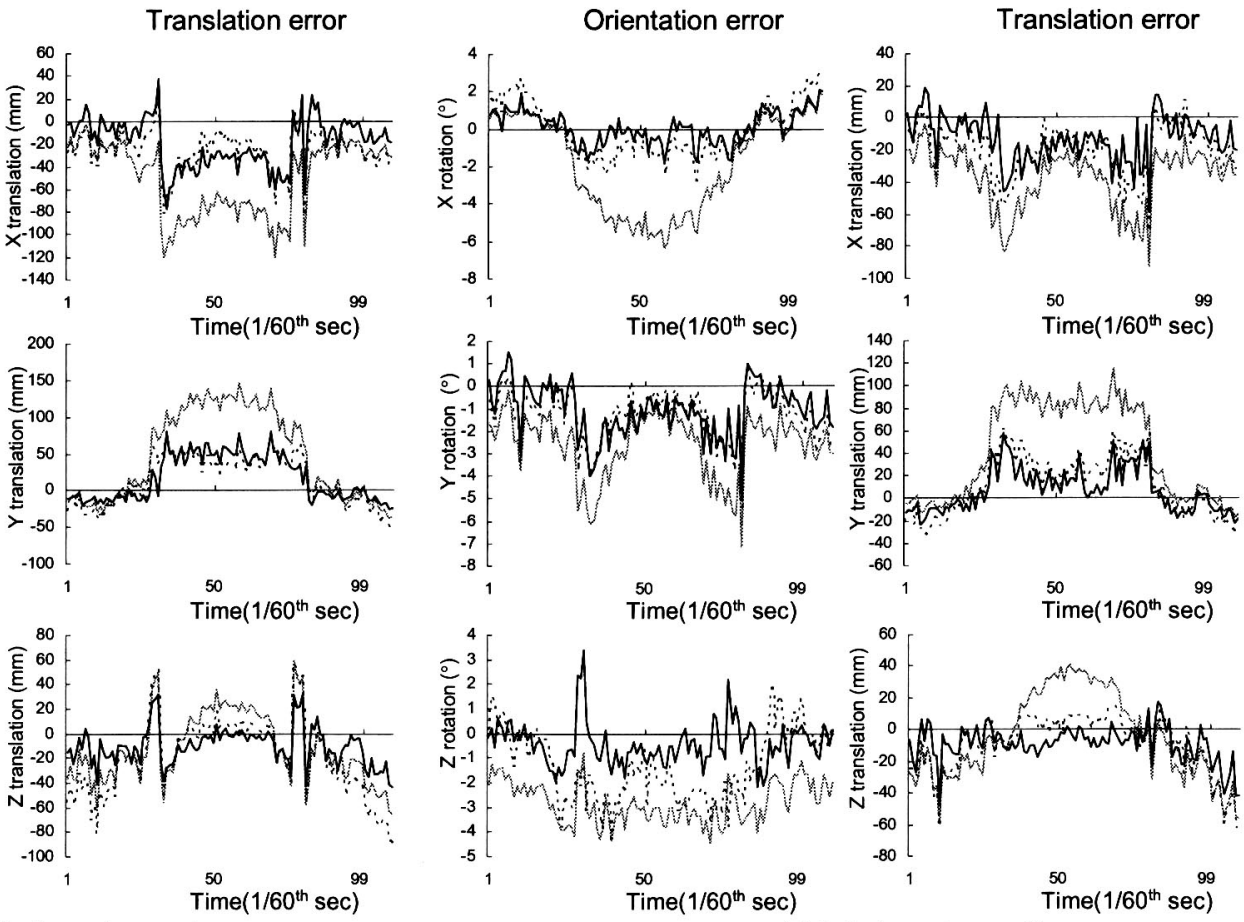

b) Marker cluster 2

Figure 3. Kinematic errors of the three methods in motion analysis of the patient (solid black: AL compensation with skin marker displacements, dashed black: AL compensation with joint angle, solid gray: without compensation) 

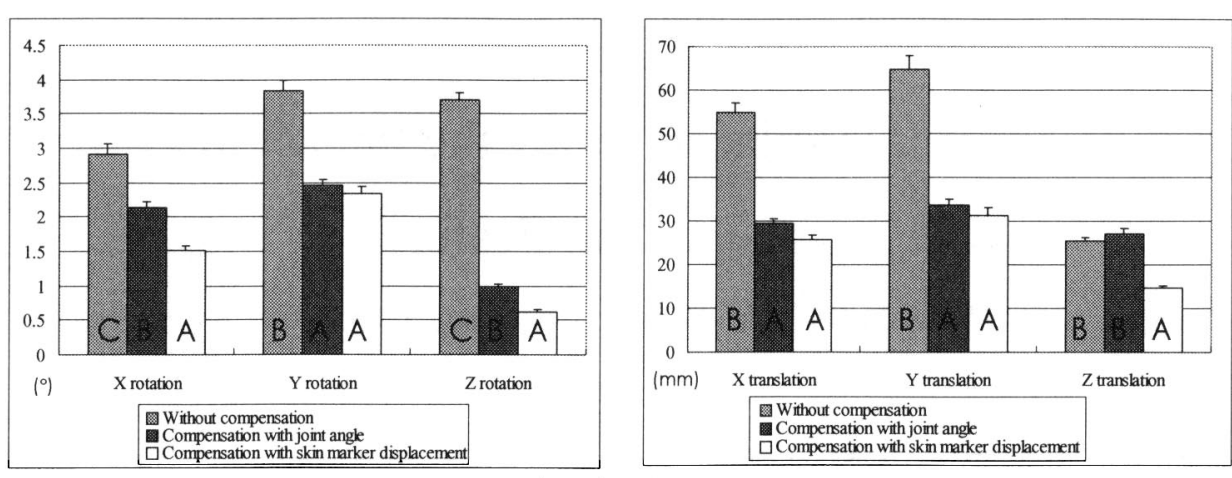

a) Marker cluster 1
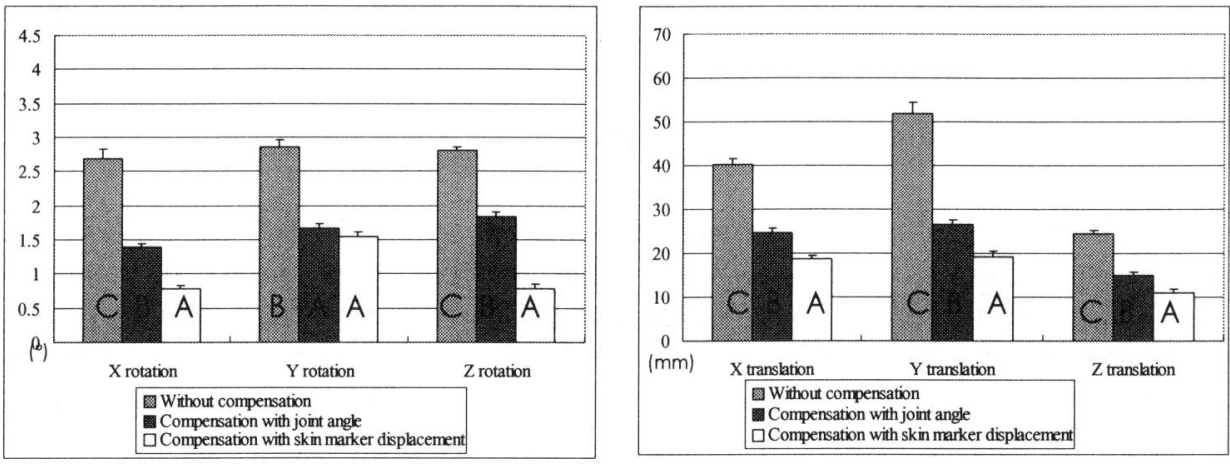

b) Marker cluster 2

Figure 8. Student-Newman-Keuls (SNK) test of motion analysis errors. The statistical groups of the errors were presented with the Alphabetic letters A, B and C.

In terms of motion analysis procedure, the two $\mathrm{AL}$ compensation methods are considerably more complex. Both require the four supplementary analysis steps: 1) identifying $\mathrm{AL}$ positions in an ad hoc motion, 2) calculating the $\mathrm{AL}$ displacement, 3) modeling $\mathrm{AL}$ displacements and 4) compensating AL position with the developed model during a motor task. The AL compensation methods should develop AL displacement models for each person through these steps. However, the benefits of the AL compensation methods outweigh the costs as shown in the validation results of the present study.

Among the two AL compensation methods, one using skin marker displacement was slightly more accurate in analyzing shank movements than the other. In the validation test, all of the errors in shank rotation and translation were more reduced with the $\mathrm{AL}$ compensation using skin marker displacement than using rotational angle. Some of differences in errors between the two methods were statistically significant, showing $25-60 \%$ reductions.

Concluding, this study proposed an alternative to the $\mathrm{AL}$ displacement modeling with joint angle of the previous dynamic calibration method. The proposed method models the AL displacement with skin marker displacement instead of joint angle and compensates AL positions more efficiently by removing the repetitive joint angle calculation in the previous method. As a result of validation test, the proposed method was effective in STA error reduction similar to the previous method. But it is necessary to validate the proposed method for various people, especially abled body.

\section{References}

[1] Cappozzo A., Catani F., Leardini A., Benedetti M.G., Della Croce U.: Position and orientation in space of bones during movement: experimental artifacts., Clinical Biomechanics, 11: 90-100, 1996

[2] Holden J.P., Orsini J.A., Siegel K.L., Kepple T.M., Gerber L.H., Stanhope S.J.: Surface movement error in shank kinematics and knee kinetics during gait., Gait \& Posture, 5: 217-227, 1997

[3] Reinschmidt C., van den Bogert A.J., Lundberg A., Nigg B.M., Murphy N., Stacoff A., Stano A.:

Tibiofemoral and tibiocalcaneal motion during walking: external vs. Skeletal markers., Gait \& Posture, 6: 98-109, 1997

[4] Lucchetti L., Cappozo A., Cappello A., Croce U.D.: Skin movement artefact assessment and compensation in the estimation of knee-joint kinematics., $J$.

Biomechanics, 31: 977-984, 1998

[5] Wu G., Siegler S., Allard P., Kirtley C., Leardini A., Rosenbaum D., Whittle M., D'Lima D.D., Cristofolini L., Witte H.: ISB recommendation on definitions of joint coordinate system of various joints for the reporting of human joint motion - part I: ankle, hip, and spine., J. Biomechanics, 35: 543-548, 2002

[6] Soderkvist I., Wedin P.: Determining the movements of the skeleton suing well-configured markers., J.

Biomechanics, 26: 1473-1477, 1993 